\title{
HOSTIGAMIENTO Y ACOSO: ACERCAMIENTO A ESTOS TIPOS DE VIOLENCIA A PARTIR DE LAS VIVENCIAS DEL PERSONAL DE LA UNIVERSIDAD AUTÓNOMA DE YUCATÁN, MÉXICO ${ }^{1}$

\author{
BULLYING AND HARASSMENT: \\ LIVED BY THE STAFF AT THE AUTONOMOUS UNIVERSITY OF YUCATÁN, MEXICO
} AN APPROACH TO THESE TYPES OF VIOLENCE FAS FROM THE EXPERIENCES
}

\author{
Leticia Janet Paredes-Guerrero² \\ Fecha de recepción: 10 de noviembre de 2018 \\ Fecha de aceptación: 30 de noviembre de 2018
}

\begin{abstract}
Resumen - La violencia en el espacio universitario es una realidad que se ha documentado en diversos estudios (Bedolla y García, 1998; Vélez Bautista y Soraya, 2013; Evangelista Tinoco y Tuñón, 2015); sin embargo, es una situación que requiere seguir documentándose y avanzando en la interpretación. Así, en el presente artículo se considera el acoso y el hostigamiento como una violencia directa, usando el modelo de violencia de Johan Galtung (1969 y 1998); se definen el hostigamiento y acoso a partir de lo establecido en la Ley General de Acceso de las Mujeres a una Vida Libre de Violencia (2007) de México, de los estudios de Roberto Castro y Verónica Vázquez García (2008), y de Araceli Mingo y Hortensia Moreno (2015), cuyos planteamientos que dan paso a las escalas de severidad que puede adquirir la violencia se retoman. Todo ello como marco de análisis para visibilizar y explicar los hallazgos sobre la violencia entre el personal de la Universidad Autónoma de Yucatán.
\end{abstract}

\section{Palabras clave:}

Hostigamiento, acoso, violencia, universidades.
Abstract - Violence in higher education institutions is a reality documented by several studies (Bedolla and García, 1998; Vélez Bautista and Soraya, 2013; Evangelista Tinoco and Tuñón, 2015); however, this is a problem that needs further documenting and advancing in its interpretation. Thus, in this paper bullying and harassment are considered forms of direct violence, using John Galtung's violence model (1969, 1998); bullying and harassment are defined according to the Mexican General Law for Women's Access to a Life Free of Violence (2007), as well as the studies by Roberto Castro and Verónica Vázquez García (2008), and by Araceli Mingo and Hortensia Moreno (2015), whose approaches -that give way to the scales of severity that violence can acquire- are revisited. All of it, as an analysis framework to visualize and explain the findings about violence among the staff of the Autonomous University o Yucatán.

\section{Keywords:}

Gender, gender violence, university.

${ }^{1}$ Artículo aceptado mediante arbitraje de pares. 


\section{Hostigamiento y acoso en espacios universitarios}

El presente trabajo muestra la presencia e intensidad o severidad del daño o sufrimiento que provocan las manifestaciones de dos tipos de violencia: el hostigamiento y el acoso, a partir del estudio de caso realizado con el personal que labora en la Universidad Autónoma de Yucatán (UADY).

Desde los albores del siglo XXI, en México comenzó a llamar la atención de académicas y académicos la presencia de dos tipos de violencia al interior de las instituciones de educación superior (IES) y en los espacios laborales, que se denominaron hostigamiento y acoso, lo cual fue motivo para que se iniciaran estudios sobre el tema. Así, podemos destacar el trabajo pionero de Patricia Bedolla y Blanca García en 1989, sobre consideraciones conceptuales en torno al hostigamiento sexual a partir de la revisión que efectuaron acerca del surgimiento del concepto de hostigamiento; los modelos explicativos surgidos respecto de la existencia de este tipo de violencia en el entorno laboral, así como los elementos que dan contenido a esta manifestación de violencia (Bedolla y García, 1989).

En 2015, Angélica Evangelista García revisó los estudios que se han elaborado en el país sobre acoso y hostigamiento durante el siglo XXI. Los resultados que obtuvo la llevaron a concluir que las investigaciones son escasas y por su enfoque las clasifica en tres tipos: 1) trabajos con una perspectiva epidemiológica y de salud pública (Rivera-Rivera et al., 2006; Castro y Casique, 2006, en Castro y Vázquez, 2008) y más recientemente desde una óptica cualitativa (Castro y Vázquez, 2008; Vázquez y Castro, 2008 y 2009); 2) estudios con líneas desde el orden jurídico (Goslinga, 2008) y 3) investigaciones de la esfera laboral (Palomino, 2012).

En cuanto a las indagaciones más nuevas sobre el hostigamiento y acoso en las universidades mexicanas, se pueden destacar las siguientes: la llevada a cabo por Vélez Bautista y Soraya (2013) en la Universidad
Autónoma del Estado de México, en la que consignan que el hostigamiento y el acoso sexual son prácticas cotidianas en las IES y su prevalencia entre las y los estudiantes se ubica entre 20 y $40 \%$; la elaborada por Valadez Ramírez y Ríos Rivera (2014), quienes han realizado estudios acerca de las percepciones de acoso y hostigamiento sexual contra las mujeres en la Universidad Nacional Autónoma de México (UNAM) y la Universidad Autónoma de México (UAM), cuyos resultados evidencian una alta frecuencia de vivencias de acoso y hostigamiento sexual por parte de las mujeres participantes en su estudio; y el trabajo de Bermúdez-Urbina (2014), en el que los docentes son quienes aparecen, en una proporción importante, como los perpetradores de la violencia psicológica y sexual al interior de las universidades.

En Yucatán, México, los estudios sobre acoso y hostigamiento son incipientes. Podemos consignar los trabajos de Burgos y Cortés (2001) sobre el acoso a mujeres meridanas; el de Cortés y Rivero (2004) sobre el mobbing y bullying; también encontramos el de Rosado y Santana (2010) acerca de la violencia en educación media superior, el efectuado por Rosado y Ortega (2014) relativo a la violencia en la narrativa de jóvenes en educación media superior y por último, el trabajo de Echeverría, Paredes, Kantún, Batún y Carrillo (2017) sobre acoso y hostigamiento sexual en estudiantes de la Universidad Autónoma de Yucatán.

El anterior recuento de estudios acerca del tema centra su atención en visibilizar la violencia en espacios educativos, laborales o comunitarios. A su vez, el presente artículo tiene como objetivo aportar a visibilizar la presencia de la violencia en el ámbito universitario y a la interpretación del hostigamiento y el acoso como dos tipos de violencia cuyas manifestaciones causan diferentes intensidades de daño o sufrimiento a quienes la viven y quienes la infligen y perpetúan el ejercicio del poder.

Para lograr el objetivo planteado se considera al acoso y al hostigamiento como una violencia directa, usando el modelo de violencia de Johan 
Galtung (1969 y 1998). Se definen hostigamiento y acoso a partir de lo establecido en la Ley General de Acceso de las Mujeres a una Vida Libre de Violencia (2007) de México, de los estudios de Roberto Castro y Verónica Vázquez García (2008) y los de Araceli Mingo y Hortensia Moreno (2015); se retoman sus planteamientos, que dan paso a las escalas de severidad que puede alcanzar la violencia y por último, teniendo como marco de análisis lo anterior, se retoman los hallazgos sobre violencia entre el personal de la UADY.

\section{La violencia}

El sociólogo noruego Johan Galtung (1969 y 1998) define violencia como una relación social cuyo fin es provocar daño o sufrimiento y limitar las potencialidades actuales o futuras de un individuo o una colectividad. Galtung distingue tres dimensiones de la violencia, que llamaremos triángulo de la violencia de género: la "directa" o "interpersonal", es decir, aquella que se presenta de manera directa entre dos o más actores concretos; la "estructural" o "indirecta", que no es ejercida por un actor específico, sino que se genera a partir de las condiciones sociales y que tiene como fundamento la distribución desigual de los recursos; y la "cultural", que es la que se basa en representaciones y elementos simbólicos sobre los sujetos. Estas tres dimensiones, argumenta Galtung (1969, pp.167-191), forman un triángulo en cuyo vértice superior se encuentra la violencia directa o interpersonal que, como la punta de un iceberg, es la parte más visible del fenómeno. No obstante, en la base de dicho triángulo se hallan la violencia estructural y la cultural que, siguiendo con la metáfora del iceberg, permanecen sumergidas en lo profundo de la sociedad y, por lo tanto, resultan poco evidentes para los sujetos, aunque pueden visibilizarse a través de la investigación y de la acción política.

Bajo este planteamiento podemos señalar que el acoso y el hostigamiento son dos tipos de violencia interpersonal, pero que tienen como base la violencia estructural y la simbólica -cultural-, que desde el análisis de la desigualdad de género, dichas violencias se basan en prescripciones, asignaciones sociales, normas y roles que colocan a hombres y mujeres en condiciones, posiciones y valoraciones asimétricas. Aunado a lo anterior se encuentran las percepciones que se interiorizan, que hacen parecer como algo "natural" y que por ende normalizan la violencia tanto para quien la ejerce como para quien la recibe, con lo que se produce el ejercicio de poder.

\section{Hostigamiento y acoso}

El hostigamiento y el acoso sexual son dos tipos de violencia interpersonal, presentes en diversos espacios, como las universidades; además, son relaciones de género desiguales que manifiestan ejercicio de poder. A partir de esta concepción se retoma la definición del acoso y el hostigamiento establecida en la Ley General de Acceso de las Mujeres a una Vida Libre de Violencia $(2007$, p. 4) de México, que en su artículo 13 "De la violencia laboral y docente" señala:

El hostigamiento "como el ejercicio del poder, en una relación de subordinación real de la víctima frente al agresor en los ámbitos laboral y/o escolar, el cual se expresa en conductas verbales, físicas o ambas, relacionadas con la sexualidad de connotación lasciva". Los elementos que forman el hostigamiento son: 1) una forma de violencia de género, 2) una clara relación asimétrica, identificándose con mayor precisión en espacios laborales y educativos; 3) una conducta de naturaleza sexual no recíproca, y toda otra conducta basada en el sexo, que afecte a la dignidad de mujeres y hombres, que resulte ingrata, irrazonable y ofensiva para quien la recibe, 4) el rechazo de una persona a esa conducta, o su sumisión a ella, se emplea explícita o implícitamente como base para una decisión que afecta algún área de la vida de esa persona, 5) es una conducta que crea un ambiente 
de trabajo intimidatorio, hostil o humillante para quien la recibe y hasta para terceras personas, 6) son conductas basadas en la coerción sexual que generan sentimientos de desagrado, que pueden expresarse a través de sensaciones de humillación, poca satisfacción personal, molestia o depresión.

La misma Ley (2007, p. 5) considera al "acoso sexual como una forma de violencia en la que, si bien no existe la subordinación, hay un ejercicio abusivo de poder que conlleva a un estado de indefensión y de riesgo para la víctima, independientemente de que se realice en uno o varios eventos". Espinosa Torres (2008, p. 13) señala que los comportamientos que se califican como acoso sexual son: a) físicos: violencia física, tocamientos, acercamientos innecesarios; b) verbales: comentarios y preguntas sobre el aspecto, estilo de vida, orientación sexual; c) no verbales: silbidos, gestos de connotación sexual, exposición de objetos pornográficos.

Tabla 1.

Características del hostigamiento y el acoso sexual

\begin{tabular}{|l|l|}
\hline \multicolumn{1}{|c|}{ Hostigamiento sexual } & \multicolumn{1}{c|}{ Acoso sexual } \\
\hline Relación real de subordinación por parte de la víctima & $\begin{array}{l}\text { No existe una relación de subordinación entre víctima y } \\
\text { agresor }\end{array}$ \\
\hline Se practica en ámbitos laborales y/o escolares & Se practica en ámbitos laborales y/o escolares \\
\hline $\begin{array}{l}\text { Se expresa en conductas verbales o físicas, o ambas, } \\
\text { relacionadas con la sexualidad de connotación lasciva }\end{array}$ & $\begin{array}{l}\text { Se expresa en el uso abusivo del poder, que conduce a un } \\
\text { estado de indefensión y de riesgo para la víctima }\end{array}$ \\
\hline No establece el número de veces de la conducta & No establece el número de veces de la conducta \\
\hline
\end{tabular}

Fuente: Instituto Nacional de las Mujeres (INMUJERES, 2009, p. 19).

El hostigamiento y el acoso sexual son expresiones de relaciones de poder directa e indirecta, ya que en la primera se establece un ejercicio de poder basado en una relación jerárquica de subordinación y en la segunda un abuso del poder basado en una relación de pares o de iguales, pero en ambas las personas que lo viven se encuentran en una posición de menor poder, más vulnerables e inseguras. Este tipo de violencia es muy frecuente que se dificulte identificarla, pues como señala Mingo (2010, p. 26), "se ejerce en formas sutiles o se ha naturalizado a través de su cotidianidad, y de usos y costumbres. Dicha dificultad se observa tanto en quienes la padecen como en quienes están presentes en el ejercicio de ésta". Es tal la sutileza de la manifestación del hostigamiento y el acoso, que produce malestares y problemas laborales, escolares, de salud, sociales, psicológicos y físicos que pocas veces son reconocidos como consecuencia de aquéllos. También existe una serie de realidades que complejizan las vivencias de la violencia; de acuerdo con Sanmartín (2012, p. 156) son: miedo personal, sentimientos de impotencia y síndrome de acomodación, pero las claves que permiten entender la perpetración de violencia son la asimetría del poder, el miedo institucionalizado, la amenaza y la sumisión, elementos que están presentes en el hostigamiento y el acoso.

\section{Escalas de severidad del hostigamiento y el acoso}

El hostigamiento y el acoso se manifiestan en prácticas y conductas como las siguientes: 1) exposición a carteles, calendarios o pantallas de computadora o de teléfono celular con imágenes de naturaleza sexual que incomoden; 2) cartas, llamadas telefónicas, correos 
electrónicos o mensajes en redes sociales y teléfonos celulares de naturaleza sexual no deseados; 3 ) miradas morbosas o gestos sugestivos que incomoden; 4) piropos, comentarios o frases de carácter sexual que molesten u ofendan; 5) insinuaciones o propuestas para tener relaciones sexuales a cambio de algo (dinero, calificaciones, objetos tecnológicos, ropa); 6) presión para aceptar invitaciones a encuentros o citas no deseados, dentro o fuera de la escuela; 7) castigos, maltrato, aislamiento, asignación de actividades que no competen a deberes escolares $u$ otras medidas disciplinarias al rechazar proposiciones sexuales; 8) contacto físico, tocamiento o manoseo sin consentimiento; 9) miedo de ser atacada/o, o abusada/o sexualmente; 10) intento de violación; 11) ser forzada/o a tener relaciones sexuales.
A las prácticas y conductas anteriores las denominaremos manifestaciones de hostigamiento y acoso. Castro y Vázquez (2008), a través de la investigación que realizaron en la Universidad Autónoma de Chapingo sobre la reproducción de la violencia, muestran que ésta va aumentando de intensidad mientras las alumnas cursan toda la licenciatura. Lo anterior lleva a considerar que no se puede suponer que las distintas expresiones de violencia ocasionan la misma proporción de daño o sufrimiento, por lo que se pueden detectar aquellas que ocasionan magnitud de daño o sufrimiento leve, moderado y grave, siendo la violencia física el punto más extremo. A partir de este planteamiento se establece la siguiente escala para conocer el nivel de severidad del hostigamiento y el acoso.

\section{Tabla 2.}

Escala de severidad del hostigamiento y el acoso

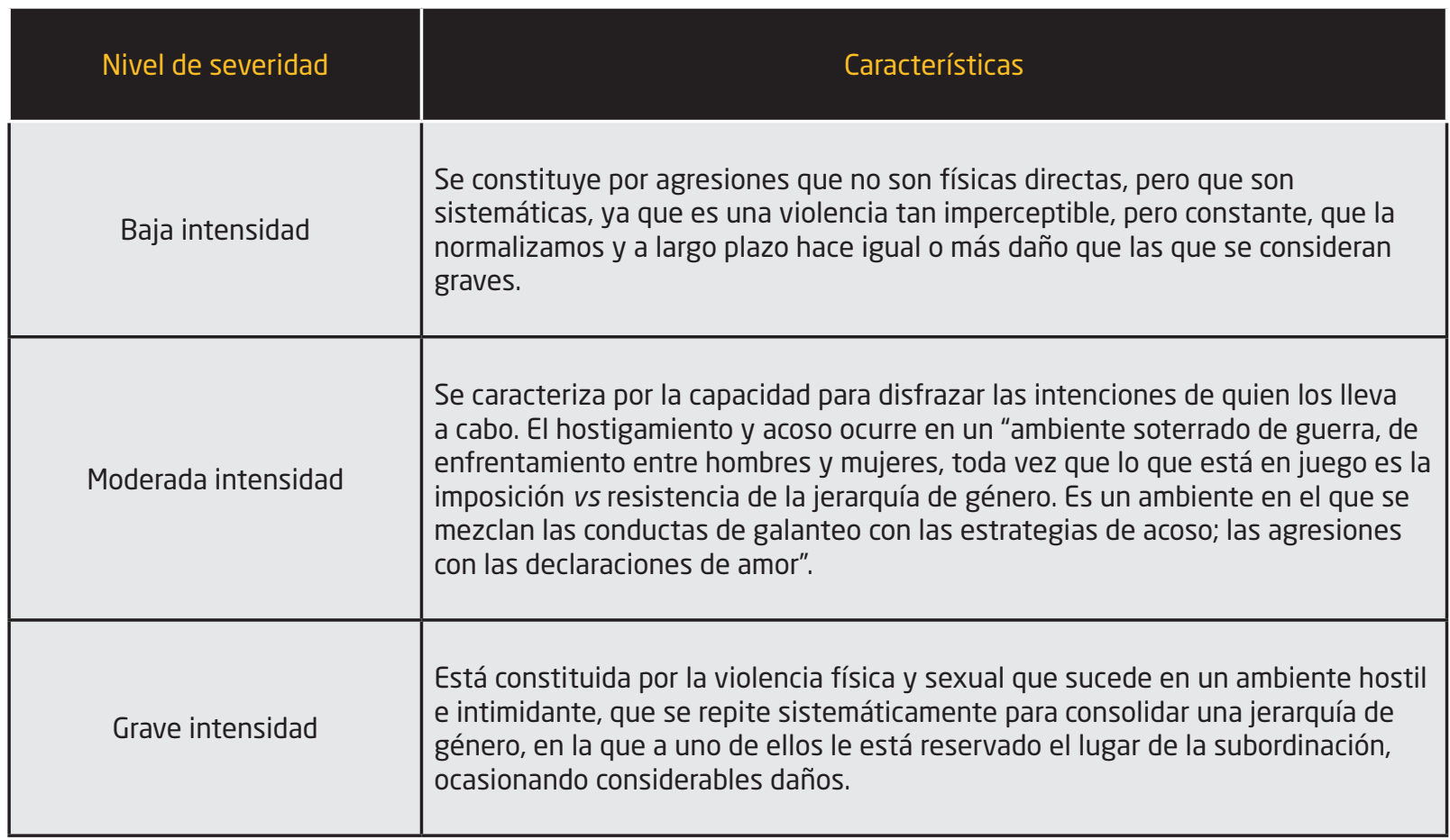

Fuente: Elaboración propia a partir del trabajo de Castro y Vázquez (2008, pp. 60, 606 y 609). 
Araceli Mingo y Hortensia Moreno (2015, p. 141) también consideran que hay evidencia (de violencia) de la "vulneración rutinaria de los derechos de muchas mujeres -y de algunos hombres- mediante prácticas que, la mayoría de las veces, o no reciben la atención debida, o por habituales pasan inadvertidas, o no reciben atención". Con base en lo anterior proponen una escala que denominan "escala del sexismo", 3 a través de ella dan cuenta que el hostigamiento y acoso son manifestaciones del sexismo y que de acuerdo con la severidad de la práctica llaman la atención, pues de otra manera son silenciadas, aceptadas y normalizadas. La siguiente tabla concentra la escala y las características de cada nivel:

\section{Tabla 3.}

Escala de severidad sexista del hostigamiento y el acoso

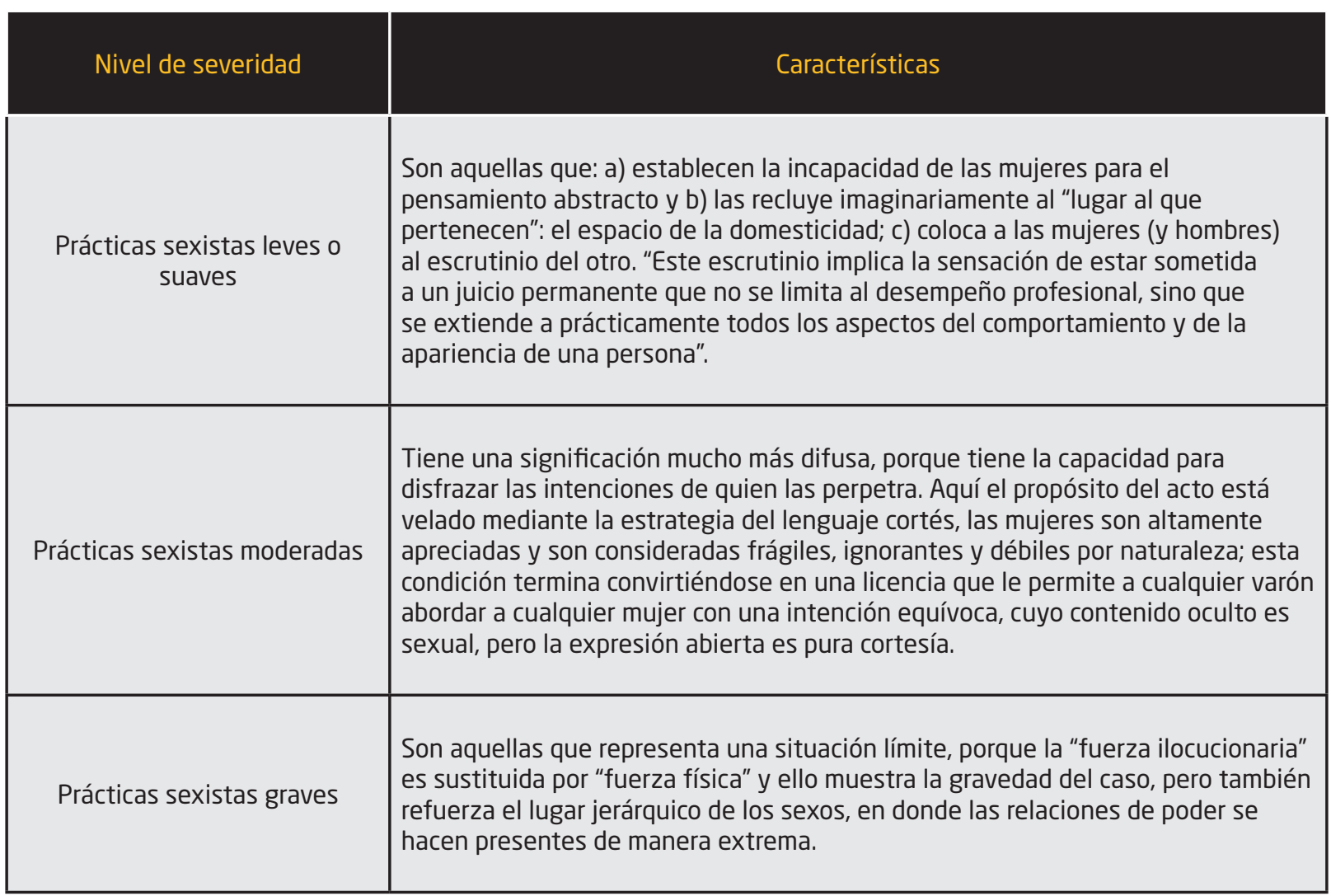

Fuente: Elaboración propia a partir del trabajo de Mingo y Moreno (2015, pp. 143-146 y 148-151).

\footnotetext{
${ }^{3}$ Las autoras señalan que: "Partimos de la idea de que el sexismo no es un hecho aislado ni excepcional en la vida de las personas, sino una práctica rutinaria, dirigida y llena de sentido social. Cuando hablamos de 'sexismo' nos referimos a percepciones y prácticas de sentido común -apuntaladas en la certidumbre de una distinción natural entre mujeres y hombres- que establecen lugares jerárquicos entre los sexos y postulan un posicionamiento de supremacía varonil. En nuestra perspectiva teórica, el sexismo es performativo porque se realiza en la acción. Es en la vida cotidiana, en la enmarañada red de las interacciones sociales, donde se manifiesta, se consuma y se reproduce" (Mingo y Moreno, 2015, pp. 141-142).
} 
Podemos decir que las escalas expuestas líneas arriba proporcionan elementos que permiten entender que las manifestaciones de violencia de hostigamiento y acoso pueden ser de distintas intensidades, pues la proporción del daño o sufrimiento es diferente. La primera no es nombrada por sus autoras, pero proponemos denominarla "progresiva", en virtud del continuo contextual en el que se encuentren quienes la viven; es decir, las manifestaciones de hostigamiento y acoso inician leves y avanzan hacia moderadas y luego a graves. A la segunda las mismas autoras la llaman "sexista", pues le dan sustento las manifestaciones de esta clase, entendido el sexismo como la desvalorización de un sexo por el otro, que se ha normalizado.
Estas escalas hacen posible clasificar las expresiones de violencia, específicamente de hostigamiento y de acoso, y tres niveles de intensidad o severidad (leve, moderado y grave), que van de forma ascendente y cuyo nivel más bajo (leve) son aquellas prácticas verbales y no verbales, seguidas (moderada) por aquellas que de manera oculta tienen un contenido sexual, pero que se disfrazan de galanteo, y en el nivel más alto (grave) se encuentra la violencia física y sexual.

Al relacionar las características de cada uno de los niveles de las escalas de severidad con las prácticas y conductas mencionadas al inicio de este apartado, se obtuvo como resultado la siguiente tabla, en la que se clasifican los grados de severidad:

\section{Tabla 4.}

Nivel de severidad con tipos de manifestaciones del hostigamiento y el acoso

Tipos de manifestaciones del hostigamiento y el acoso

Exposición a carteles, calendarios o pantallas de computadora o de teléfono celular con imágenes de naturaleza sexual que incomoden

Cartas, llamadas telefónicas, correos electrónicos o mensajes en redes sociales y teléfonos celulares, de naturaleza sexual no deseados

Miradas morbosas o gestos sugestivos que incomoden

Piropos, comentarios o frases de carácter sexual que molesten u ofendan

Insinuaciones o propuestas para tener relaciones sexuales a cambio de algo (dinero, calificaciones, cosas, objetos tecnológicos, ropa)

Presión para aceptar invitaciones a encuentros o citas no deseados, dentro o fuera de la escuela

Castigos, maltrato, aislamiento, asignación de actividades que no competen a deberes escolares u otras medidas disciplinarias al rechazar proposiciones sexuales

Contacto físico, tocamiento o manoseo sin consentimiento

Miedo de ser atacada/abusada sexualmente

Intento de violación

Coaccionada/forzada/o a tener relaciones sexuales

Fuente: Paredes, Kantún, Batún, España, Echeverría y Carrillo (2018).
Nivel de severidad

Leve

Moderado

Grave 
A partir de los planteamientos plasmados, a continuación se analizan los hallazgos sobre hostigamiento y acoso en el personal que labora en la Universidad Autónoma de Yucatán.

\section{Presencia de hostigamiento y acoso en la Universidad Autónoma de Yucatán}

Para este trabajo se retoman los resultados de la investigación "Equidad de género en la Universidad
Autónoma de Yucatán" (Paredes, 2016), en la cual obtuvimos información a través de una encuesta aplicada a $78.7 \%$ de la población universitaria, integrada por personal docente (62\%), personal administrativo y personal manual (36.8\%). En el siguiente cuadro vemos la distribución por sexo.

\section{Cuadro 1.}

Personal encuestado por sexo

\begin{tabular}{|c|c|c|c|c|}
\hline \multirow{2}{*}{ Tipo de personal } & \multicolumn{2}{|c|}{ Hombres } & \multicolumn{2}{c|}{ Múeres } \\
\cline { 2 - 5 } Académicos & 713 & 63.71 & 552 & 55.87 \\
\hline Administrativos & 178 & 15.90 & 369 & 37.34 \\
\hline Manuales & 228 & 20.37 & 67 & 6.78 \\
\hline Total & 1,119 & 100 & 988 & 100 \\
\hline
\end{tabular}

Fuente: Elaboración con base en Paredes, 2016.

En la encuesta aplicada al personal se plantearon tres esferas de estudio, la primera fue la inserción al espacio universitario, la segunda el clima laboral en la universidad y la tercera las políticas universitarias. De estas tres, se advirtió que en los resultados de la segunda dimensión se visibilizó la presencia de hostigamiento y acoso entre quienes trabajan en la universidad; así, de 2,718 personas encuestadas, 1,807 -es decir, 66.5\%-manifestó haber tenido algún evento de hostigamiento o acoso.

Si bien en general el hostigamiento y el acoso se definen a partir de lo que señala la Ley General de Acceso de las Mujeres a una Vida Libre de Violencia, para el caso particular de esta universidad se hacen precisiones, quedando de la siguiente manera: el hostigamiento se suscita cuando quien lo ejerce ostenta mayor orden jerárquico en la estructura universitaria y el acoso cuando la persona que lo perpetra está en el mismo nivel jerárquico que quien lo padece, pero el abuso de poder ocurre por condición de desigualdad de género.

De las manifestaciones de hostigamiento o acoso que señala la literatura, el personal universitario expresó haber vivido seis : 1) comentarios no deseados acerca de la apariencia física; 2) llamadas telefónicas, correos electrónicos y/o mensajes de naturaleza sexual no deseados por parte del personal de la institución; 3) bromas, comentarios o preguntas incómodas sobre la vida sexual o amorosa; 4) presiones para aceptar invitaciones no deseadas fuera del trabajo; 5 ) roces 0 contacto físico no deseado; 6) uso de la fuerza física o presión psicológica para tener relaciones sexuales.

El sector que ha enfrentado con mayor frecuencia los comportamientos antes señalados han sido 
las mujeres y de esta población, en particular las trabajadoras manuales fueron quienes sufrieron más experiencias de violencia. También se registraron acciones que se presentaron tanto en mujeres como en hombres y hubo algunas que sólo las experimentaron los varones. En la siguiente tabla describimos por sexo y porcentaje 4 las expresiones de violencia que ha vivido el personal académico, administrativo y manual.

\section{Tabla 5.}

Manifestaciones de violencia vividas por mujeres y hombres que laboran en la UADY

\section{Las manifestaciones de violencia más frecuentes vividas por mujeres son:}

1. Llamadas telefónicas, correos electrónicos y/o mensajes de naturaleza sexual no deseados: quienes más las han experimentado fueron las trabajadoras manuales, con $6 \%$.

2. Bromas, comentarios o preguntas incómodas sobre la vida sexual o amorosa: $4 \%$ de las académicas, $17 \%$ de las administrativas y $26.87 \%$ de las manuales la han sufrido.

\section{Las manifestaciones de violencia más frecuentes vividas por ambos sexos son:}

1. Comentarios acerca de la apariencia: de los tres grupos de trabajadores y trabajadoras, quienes afirmaron haberlos recibido con mayor frecuencia fueron las mujeres que laboran como manuales, con 40.30\%. En tanto que las académicas y administrativas los han experimentado con menor frecuencia, 15\%. Los hombres también han recibido dichos cometarios, pues $15 \%$ de los administrativos lo señalaron, al igual que $19 \%$ de los trabajadores manuales.

2. Presiones para aceptar invitaciones no deseadas fuera del trabajo: quienes más las han experimentado son las trabajadoras manuales, con $12 \%$; las administrativas con $5 \%$ y los hombres académicos en $2.24 \%$.

3. Roces o contacto físico no deseado: los académicos dijeron haber tenido eventos de este tipo en $24.82 \%$, seguido de las trabajadoras manuales con $19.40 \%$ y finalmente $11.38 \%$ de las mujeres administrativas.

La manifestación de violencia más frecuente que vivieron los hombres es:

1. Uso de fuerza física o presión psicológica para tener relaciones sexuales: esta manifestación de violencia fue referida pocas veces, pero quienes lo hicieron fueron los empleados administrativos, con 1,12\%, y .88\% de los manuales.

La tabla anterior visibiliza la presencia de hostigamiento y acoso entre el personal que labora en la universidad, pero también se puede observar que de las seis manifestaciones de violencia que dijeron haber sufrido, las trabajadoras manuales, quienes en el orden jerárquico ocupan el nivel más bajo, aseguraron haber vivido cinco (con diferente frecuencia). Las mujeres administrativas, que ostentan un nivel intermedio en el orden laboral, afirmaron que experimentaron cuatro; en tanto que las académicas, quienes ocupan las primeras posiciones del orden jerárquico en la estructura laboral, señalaron que únicamente han sufrido dos de los seis tipos de manifestación de violencia.
En cuanto a los hombres, tanto los académicos como los administrativos y manuales dijeron haber sufrido cuatro de las seis formas de violencia. Aquí lo interesante es que lo que más vivieron los académicos está vinculado con violencia física y sexual.

Los resultados del estudio mostraron que en la población de mujeres los tipos de violencia que padecen recurrentemente son la verbal y la psicológica, pero también se percibió que la diversidad de expresiones de hostigamiento y acoso que han recibido se relaciona con la posición que ocupen en el orden jerárquico de la universidad. En cuanto a los varones, los tipos de violencia que más mencionaron fue la física y sexual, y llama la atención su poca relación con el orden jerárquico.

${ }^{4}$ Todos los porcentajes se calcularon a partir del total de cada tipo de población encuestada y de las manifestaciones de violencia. En los cuadros se presentan sin jerarquía alguna. 
Esta diferencia nos lleva a concluir que son distintas las violencias vividas en cada sexo, que el género es una condición importante para que se manifiesten y que el hostigamiento y el acoso se complejiza en el caso de las mujeres, pues hay que relacionarla con la violencia institucional dada a partir del orden jerárquico de la estructura laboral, que las coloca en posiciones de mayor vulnerabilidad.

\section{Escala de severidad de hostigamiento 0 acoso en la UADY}

Visibilizar el hostigamiento y acoso entre quienes laboran en la universidad y conectarlos con las escalas de severidad que señalamos líneas arriba (progresiva y sexista) permitió dar cuenta que las seis manifestaciones expresadas por el personal se distribuyen de la siguiente forma:

a. Dos las podemos ubicar en el nivel leve, ya que una cumple con las características de este nivel de la escala sexista y la otra con la de la escala progresiva: la primera que colocamos en el grado leve de la escala sexista son los "comentarios no deseados acerca de la apariencia", la cual -de acuerdo con la escala de sexismo de Mingo y Moreno- implica poner a las mujeres (y hombres) bajo el escrutinio del otro. "Este escrutinio implica la sensación de estar sometida a un juicio permanente que no se limita al desempeño profesional, sino que se extiende a prácticamente todos los aspectos del comportamiento y de la apariencia de una persona" (Mingo y Moreno, 2015, pp.143-45). Dicha manifestación la han experimentado trabajadoras manuales, administrativas y académicas. La segunda que colocamos en este peldaño son las "llamadas telefónicas, correos electrónicos y/o mensajes de naturaleza sexual no deseados, de personal de la institución", que según la escala progresiva de Castro y Vázquez (2008, p. 60), "son agresiones que no son físicas directas, pero que sí son sistemáticas, ya que es una violencia tan imperceptible, pero constante, que la normalizamos y a largo plazo hace igual o más daño que las que se consideran graves". Quienes más vivencias tienen de este tipo son las trabajadoras administrativas y manuales.

b. Otras dos expresiones se ubican en el nivel moderado y ambas cumplen con las características de la escala progresiva: una refiere a las "bromas, comentarios o preguntas incómodas sobre la vida sexual o amorosa" y a otra a "presiones para aceptar invitaciones no deseadas fuera del trabajo". De acuerdo con la escala progresiva de Castro y Vásquez (2008, p. 606), “Tiene como característica la capacidad para disfrazar las intenciones de quien lo perpetra. El hostigamiento y acoso se da en un ambiente soterrado de guerra, de enfrentamiento entre hombres y mujeres, toda vez que lo que está en juego es la imposición vs resistencia de la jerarquía de género. Es un ambiente en el que se mezclan las conductas de galanteo con las estrategias de acoso; las agresiones con las declaraciones de amor". El personal universitario que ha sufrido la primera manifestación son las académicas, administrativas y manuales, y quienes han experimentado la segunda son las que trabajan como administrativas y manuales, así como los académicos.

c. Las dos últimas demostraciones de violencia las colocamos en el nivel grave y ambas tienen las características de la escala del sexismo: la primera son "roces o contacto físico no deseado", la segunda es "uso de la fuerza física o presión psicológica para tener relaciones sexuales". Según la escala de sexismo de Mingo y Moreno (2015, pp. 148-151), representa una situación límite porque la "fuerza ilocucionaria" es sustituida por "fuerza física" y ello muestra la gravedad del caso, pero también refuerza el lugar jerárquico de los sexos, en donde las relaciones de poder se hacen presentes de manera extrema. El personal de la universidad que dijo haber vivido estas expresiones de violencia, en el primer caso, son mujeres administrativas y manuales y varones académicos. $Y$ quienes sufrieron el segundo tipo son hombres administrativos y manuales. 
En la siguiente tabla sintetizamos lo descrito en líneas anteriores.

\section{Tabla 6.}

Relación entre las manifestaciones de hostigamiento y acoso y las escalas de severidad entre el personal académico, administrativo y manual de la UADY

\begin{tabular}{|c|c|c|c|}
\hline $\begin{array}{c}\text { Manifestaciones de } \\
\text { hostigamiento y } \\
\text { acoso }\end{array}$ & $\begin{array}{c}\text { Escalas de intensidad } \\
\text { de HAS }\end{array}$ & Mujeres & Hombres \\
\hline $\begin{array}{c}\text { Comentarios no } \\
\text { deseados acerca de la } \\
\text { apariencia }\end{array}$ & $\begin{array}{c}\text { Leve } \\
\text { Son aquellas que: a) establecen la incapacidad } \\
\text { de las mujeres para el pensamiento abstracto, } \\
\text { b) recluyen imaginariamente a la mujer en } \\
\text { el "lugar al que pertenece": el espacio de la } \\
\text { domesticidad, y c) colocan a las mujeres (y } \\
\text { hombres) bajo el escrutinio del otro. }\end{array}$ & $\begin{array}{c}\text { Académicas, } \\
\text { administrativas y } \\
\text { manuales }\end{array}$ & \\
\hline $\begin{array}{l}\text { Llamadas telefónicas, } \\
\text { correos electrónicos } \\
\text { y/o mensajes de } \\
\text { naturaleza sexual no } \\
\text { deseados por parte } \\
\text { de personal de la } \\
\text { institución } \\
\end{array}$ & $\begin{array}{c}\text { Leve } \\
\text { Se compone de agresiones que no son físicas } \\
\text { directas, pero que sí son sistemáticas, ya } \\
\text { que es una violencia tan imperceptible, pero } \\
\text { constante, que la normalizamos y a largo } \\
\text { plazo hace igual o más daño que las que se } \\
\text { consideran graves. }\end{array}$ & Manuales & \\
\hline $\begin{array}{l}\text { Bromas, comentarios } \\
\text { o preguntas } \\
\text { incómodas sobre la } \\
\text { vida sexual o amorosa }\end{array}$ & $\begin{array}{c}\text { Moderado } \\
\text { Tiene como característica la capacidad } \\
\text { para disfrazar las intenciones de quien lo } \\
\text { perpetra. El hostigamiento y acoso suceden } \\
\text { en un "ambiente soterrado de guerra, de } \\
\text { enfrentamiento entre hombres y mujeres, } \\
\text { toda vez que lo que está en juego la } \\
\text { imposición Vs resistencia de la jerarquía de } \\
\text { género. Es un ambiente en el que se mezclan } \\
\text { las conductas de galanteo con las estrategias } \\
\text { de acoso; las agresiones con las declaraciones } \\
\text { de amor". }\end{array}$ & $\begin{array}{c}\text { Académicas, } \\
\text { administrativas y } \\
\text { manuales }\end{array}$ & \\
\hline $\begin{array}{c}\text { Presiones para } \\
\text { aceptar invitaciones } \\
\text { no deseadas fuera del } \\
\text { trabajo }\end{array}$ & $\begin{array}{c}\text { Moderado } \\
\text { Se distingue por la capacidad para disfrazar } \\
\text { las intenciones de quien lo lleva a cabo, como } \\
\text { en el caso anterior. }\end{array}$ & $\begin{array}{c}\text { Administrativas y } \\
\text { manuales }\end{array}$ & Académicos \\
\hline $\begin{array}{l}\text { Roces o contacto } \\
\text { físico no deseado }\end{array}$ & $\begin{array}{c}\text { Grave } \\
\text { Son aquellas que representan una situación } \\
\text { límite, porque la "fuerza ilocucionaria" es } \\
\text { sustituida por "fuerza física" y ello muestra } \\
\text { la gravedad del caso, pero también refuerza } \\
\text { el lugar jerárquico de los sexos, en donde las } \\
\text { relaciones de poder se hacen presentes de } \\
\text { manera extrema. }\end{array}$ & $\begin{array}{c}\text { Administrativas y } \\
\text { manuales }\end{array}$ & Académicos \\
\hline $\begin{array}{c}\text { Uso de la fuerza física } \\
\text { o presión psicológica } \\
\text { para tener relaciones } \\
\text { sexuales }\end{array}$ & $\begin{array}{l}\text { Grave } \\
\text { Sus características son las mismas que la } \\
\text { descripción anterior. }\end{array}$ & & $\begin{array}{c}\text { Administrativos y } \\
\text { manuales }\end{array}$ \\
\hline
\end{tabular}

Fuente: Elaboración propia. 
El análisis de la relación de las manifestaciones de hostigamiento y acoso que se presentan entre el personal de la UADY y las escalas de intensidad o severidad de la violencia propuestas en los estudios de Mingo y Moreno (2015) y de Castro y Vázquez (2008) permite afirmar lo siguiente: si bien las violencias colocadas en el nivel leve son parte de las prácticas sexistas que buscan mantener el orden social de desigualdad de género como conductas "naturales" de los sexos, también son expresiones que al pasar desapercibidas constituyen el primer peldaño para ejercer violencia con mayor severidad.

En cuanto a las manifestaciones colocadas en el nivel moderado, se puede decir que son prácticas tan sutiles que no nos damos cuenta de que aquellas expresiones que pasaban como leves adquieren mayor seriedad y se disfrazan bajo diversos tipos de relaciones, como por ejemplo la laboral, amorosa, la de competencia, entre otras. Por último, las ubicadas en el nivel grave son prácticas sexistas que buscan "mantener" a cada sexo en la posición de género que socialmente se les ha asignado y el ejercicio de poder es extremo.

\section{Conclusiones}

Finalmente se puede señalar que el hostigamiento y el acoso son dos tipos de violencias interpersonales, pero que tienen como base la violencia estructural y la simbólica -cultural-, basadas en relaciones de poder directas e indirectas. De este modo, el hostigamiento pone en marcha un ejercicio de poder directo a partir de la jerarquía establecida entre los sexos y entre los géneros, y en el acoso un abuso del poder indirecto que se hace presente en la relación instituida entre pares, en donde la desigualdad de género resulta muy evidente. En ambos casos, las personas que sufren la violencia se encuentran en una posición de menor poder, más vulnerables e inseguras, a diferencia de quien la inflige, pues tiene más poder, mejor posición y más seguridad. Estos tipos de violencias pueden expresarse verbalmente, no verbalmente, psicológica, física y sexualmente, pero el nivel de daño o sufrimiento que ocasionan alcanza proporciones diferentes.
Teniendo como base que no se puede generalizar el grado de daño o sufrimiento que provocan las diversas expresiones de violencia es como se puede entender que se haga referencia a diversos niveles de severidad o intensidad de ellas. Y también permite dar cuenta que los trabajos de Castro y Vázquez (2008) y de Mingo y Moreno (2015) nos brindan dos modelos para clasificar e interpretar dichas manifestaciones de acuerdo con determinadas características y colocarlas en algún nivel severidad, que puede ser leve, moderado o grave.

Los hallazgos sobre violencia entre el personal docente y administrativo obtenidos de la investigación "La equidad de género en la Universidad Autónoma de Yucatán" se clasificaron a partir de la proporción del daño o sufrimiento que causaban en quienes la habían vivido en leves, moderadas y graves. Además, se pude interpretar que el trasfondo de violencia directa del hostigamiento y el acoso es la violencia estructural y simbólica, que se materializa en las posiciones que se ocupan en la estructura laboral, en las prácticas sexistas y en los límites y resistencias para aceptar el ejercicio del poder, entre otras.

Siguiendo con este orden de ideas, se concluye que las expresiones de violencia halladas en el personal de la universidad son prácticas sexistas que buscan mantener el orden social de desigualdad de género, como conductas "naturales" de los sexos, prácticas sutiles que no advertimos cuando van progresando respecto de la afectación que ocasionan, al estar disfrazadas de relaciones laborales, amorosas y de competencia, entre otras; son actitudes sexistas que buscan "mantener" a cada sexo en la posición de género que socialmente se le ha asignado, a través del ejercicio de poder extremo. Para finalizar, es posible señalar que las manifestaciones de hostigamiento y acoso provocan mal o sufrimiento en diferentes intensidades o proporciones para quienes lo viven, y para quienes lo perpetúan significa reproducir y conservar el ejercicio del poder. 


\section{Referencias}

Bedolla, P. y García, B. (1989). Consideraciones conceptuales en torno al hostigamiento sexual. En Estudios de género y feminismo I (pp. 175-182). México: Ed. UNAM /Fontamara.

Bermúdez-Urbina, F. M. (2014). Aquí los maestros ya no pegan porque ya no se acostumbra. Expresiones de la violencia hacia las mujeres en la Universidad de Ciencias y Artes de Chiapas. Península, IX, 15-40.

Castro, R. y Vázquez García, V. (2008). La Universidad como espacio de reproducción de la violencia de género. Un estudio de caso en la Universidad Autónoma Chapingo, México. Estudios Sociológicos, XXVI(3), 587- 616.

Cortés Camarillo, G. y Rivero Evia, J. (2010). Acoso moral: una sutil derivación de la violencia. Breves notas sobre el mubbing y el bullyng y su relación con el derecho a la intimidad. Disponible en www. derecho.uady.mx/tohil/rev24/acosomoral.pdf

Burgos Fajardo, R. J. y Cortés Camarillo, G. (2001). Acoso sexual: naturaleza y significado para mujeres jóvenes y adultas en la ciudad de Mérida, Yucatán. Revista Educación y Ciencia, 5(23), Nueva época, 21-28.

Echeverría Echeverría, R., Paredes Guerrero, L., Kantún Chim, M. D., Batún Cutz, J. L. y Carrillo Trujillo, C. D. (2017). Acoso y Hostigamiento sexual en estudiantes de la UADY: un acercamiento cuantitativo. Revista Enseñanza e Investigación en Psicología, 22(1), 15-26.

Espinosa Torres, P. (2008). El acoso y hostigamiento en México. Una forma de violencia silenciosa en los centros de trabajo. Revista Sororidad, 3, 12-16.

Evangelista García, A., Tinoco Ojanguren, R. y Tuñón Pablos, E. (2015). Violencia escolar en ámbitos de educación superior y de posgrado. Estado de la cuestión. Ponencia presentada en el XI Coloquio Nacional de la Red de Estudios de Género del Pacífico Mexicano.

Galtung, J. (1969). Violence, Peace and Peace Research. Journal of Peace Research, 6(3), 167-191.

Galtung, J. (1998). Tras la violencia, 3R: reconstrucción, reconciliación, resolución.
Afrontando los efectos visibles e invisibles de la guerra y la violencia. Bilbao: Bakaeazl GernikaGogratuz.

Instituto Nacional de las Mujeres (INMUJERES). (2009). Protocolo de intervención para casos de hostigamiento y acoso sexual. México: autor.

Ley General de Acceso de las Mujeres a una Vida Libre de Violencia. (2007). Disponible en http://www. diputados.gob.mx/LeyesBiblio/pdf/LGAMVLV.pdf

Mingo, A. y Moreno, H. (2015). El ocioso intento de tapar el sol con un dedo: violencia de género en la universidad. Revista Perfiles Educativos, XXXVII(148), 138-155.

Mingo, A. (2010). Ojos que no ven... Violencia escolar y género. Perfiles Educativos, XXXII(130), 25-48.

Palomino, F. (2012). Acoso sexual en México: análisis y propuestas. En-claves del Pensamiento, VI(12), 133-157.

Paredes Guerrero, L., Kantún Chim, M. D., España Paredes, P., Batún Cutz, J. L., Echeverría, R. y Carrillo Trujillo, C. D. (2018). Estrategia para identificar Hostigamiento y Acoso sexual en la Universidad Autónoma de Yucatán. Cuaderno de Investigación UADY

Paredes Guerrero, L. (2016). Equidad de Género en la Universidad Autónoma de Yucatán. Mérida: Ediciones Universidad Autónoma de Yucatán.

Rosado, C. y Ortega, O. (2014). Narrativas jóvenes del Estado de Yucatán: entre la palabra y la violencia. La ventana. Revista de Estudios de Género, 5(40), 213-253.

Rosado Rosado, G. y Santana Rivas, L. (2010). Violencia y discriminación de género entre jóvenes de educación media superior en Mérida, Yucatán. México: Instituto para la Equidad de Género en Yucatán.

Sanmartín Esplugues, J. (2012). Claves para entender la violencia en el siglo XXI. Ludus Vitalis, XX(38), 145-160.

Valadez Ramírez, A. y Ríos Rivera, L. A. (2014). Percepciones de acoso y hostigamiento sexual contra las mujeres: un estudio exploratorio. Revista Electrónica de Psicología Iztacala, 17(2), 624-645. 
Vázquez, V. y Castro, R. (2008). ¿Mi novio sería capaz de matarme? Violencia en el noviazgo entre adolescentes de la Universidad Autónoma Chapingo, México. Revista Latinoamericana de Ciencias Sociales, Niñez y Juventud, 6(2), 709-738.

Vázquez, V. y Castro, R. (2009). Masculinidad hegemónica, violencia y consumo de alcohol en el medio universitario. Revista Mexicana de Investigación Educativa, 14(42), 701-719.

Vélez Bautista, G. y Soraya Munguía, K. (2013). Análisis, prevención y atención del hostigamiento y el acoso escolar y sexual hacia las y los estudiantes: Caso de la Universidad Autónoma del Estado de México. Conferencia dictada en el XXIX Congreso ALAS Chile "Crisis y Emergencias Sociales en América Latina". Disponible en https://www.researchgate. net/publication/315114077_Analisis_ prevencion_y_atencion_del_hostigamiento_y_ el_acoso_escolar_y_sexual_hacia_las_y_los_ estudiantes_Caso_de_la_Universidad_Autonoma_ del_Estado_de_Mexico

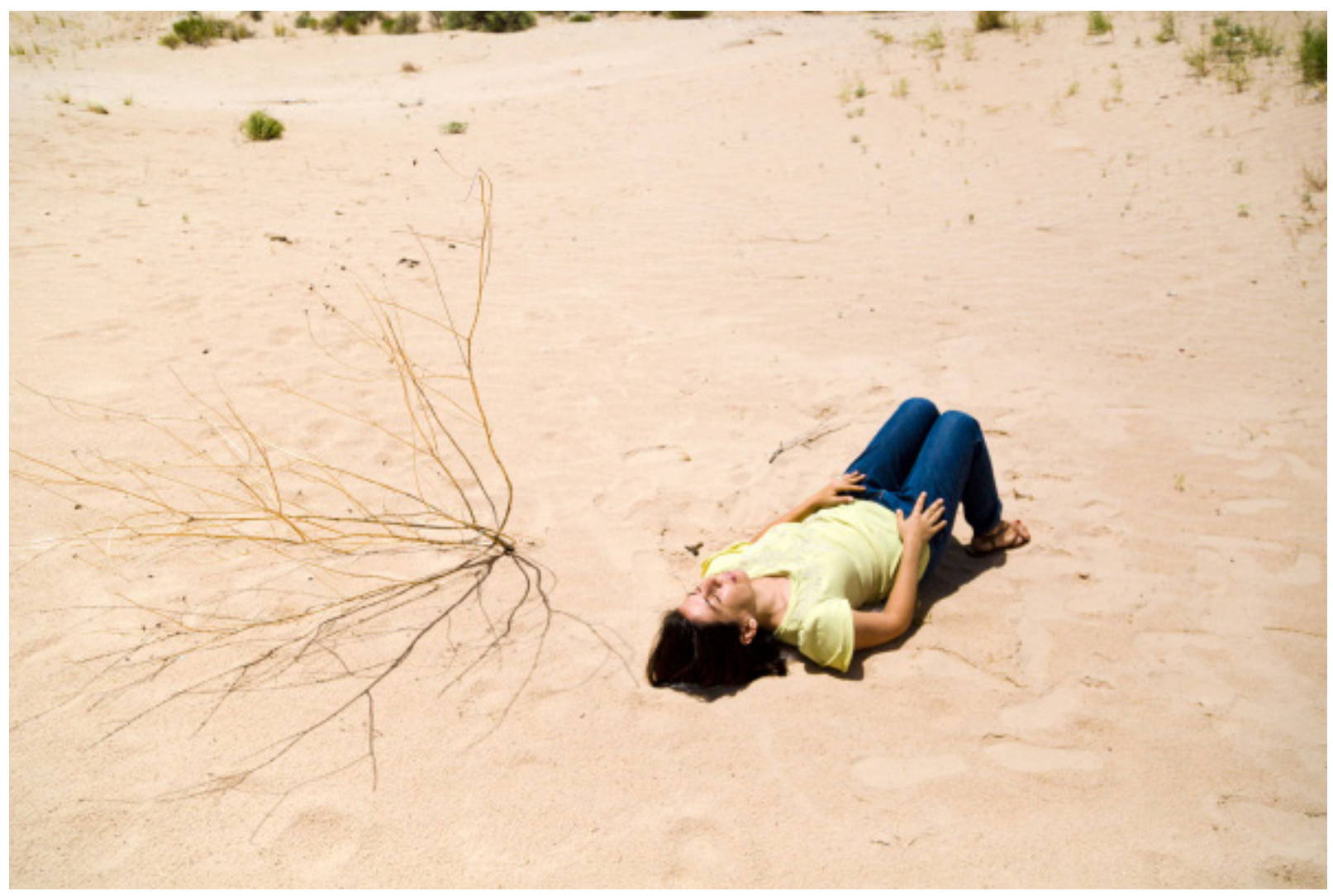

Emilia, Desierto de Chihuahua, México, 2015 